\title{
Dynamics of Shallow Water Waves with Various Boussinesq Equations
}

\author{
H. Kumar ${ }^{a, *}$ A. Malik ${ }^{b}$, M. Singh Gautam ${ }^{c}$ And F. Chand ${ }^{d}$ \\ ${ }^{a}$ Department of Physics, Dr. B.R. Ambedkar Institute of Technology, Port Blair-744103, India \\ ${ }^{b}$ Department of Physics, Chaudhary Bansi Lal University, Bhiwani-127021, India \\ ${ }^{c}$ Department of Physics, Indus Degree College, Jind-126102, India \\ ${ }^{d}$ Department of Physics, Kurukshetra University, Kurukshetra-136119, India
}

(Received September 18, 2016; in final form January 16, 2017)

\begin{abstract}
Attempt has been made to construct the solitary waves and shock wave solutions or domain walls (in higher dimension) for various Boussinesq equations. The method of undetermined coefficients have been used to explore the exact analytical solitary waves and shock wave solutions in terms of bell-shaped sech ${ }^{p}$ function and kinkshaped $\tanh ^{p}$ function for the considered equations. The Boussinesq equation in the $(1+1)$-dimensional, the $(2+1)$-dimensional and the $(3+1)$-dimensional equations are studied and the parametric constraint conditions and uniqueness in view of both solitary waves and shock wave solutions are determined. Such solutions can be valuable and desirable for explaining some nonlinear physical phenomena in nonlinear science described by the Boussinesq equations. The effect of the varying parameters on the development of solitary waves and shock wave solutions have been demonstrated by direct numerical simulation technique.
\end{abstract}

DOI: 10.12693/APhysPolA.131.275

PACS/topics: Solitons, exact solutions, Boussinesq equation

\section{Introduction}

The investigation of soliton solutions to nonlinear evolution equations (NLEEs) plays a pivotal role in the diversified nonlinear physical phenomena. The various solitary wave phenomena are observed in plasmas physics, fluid dynamics, fiber-optic communications, nonlinear photonic crystals, chemical physics, the Bose-Einstein condensates, nuclear physics, crystal lattice vibrations, biological systems, DNA molecule and energy transport in proteins $[1,2]$. The observations of solitons in various physical systems uncover many exciting problems from both central and applied points of view. Solitary waves are wave packets which travel in nonlinear dispersive media and retain their stable wave forms due to the exact dynamical counterbalancing between the nonlinear and the dispersive effects. The basic strategies one may adopt to predict, control and quantify the basic features of a physical system is to model the system in terms of mathematical equations, which are usually nonlinear and then find exact analytic solutions of such nonlinear physical model equations using some suitable methods. The exact solutions, if exist, of those nonlinear equations facilitate us to well understand the mechanism of the complicated dynamical processes exhibited by these nonlinear evolution equations.

This paper emphases on integrability aspect of the Boussinesq equations to retrieve solitary wave solution, it must be noted that there are several other approaches to obtain solitary wave solutions and other forms of

*corresponding author; e-mail: hkkhatri24@gmail.com waves such as cnoidal waves, snoidal waves and singular solitons. In recent years, many powerful and efficient integration techniques have been proposed to construct exact travelling wave solutions of NLEEs [1-27].

The dynamics of shallow water waves that are realized in various places like lakes, sea beaches and rivers are governed by the Boussinesq equation. The Kortewegde Vries equation that models shallow water waves is certainly very well known. Nevertheless, the Boussinesq equation provides a much superior approximation to such waves. Among the NLEEs, the Boussinesq equation has been resulting in order to describe the long waves transmitting on the surface of shallow water [28-31]. The Boussinesq-like equations also appear in many physical phenomena, such as electromagnetic waves in nonlinear dielectrics, one-dimensional nonlinear lattice waves, ion sound waves in plasma, and oscillations in a nonlinear string. The Boussinesq approximation is an valid approximation in hydrodynamics for weakly nonlinear and fairly long water waves. In this paper, we emphasized on obtaining the solitary waves and shock wave solutions of the Boussinesq equations. For both cases, the necessary constraint conditions are retrieved.

In this work, we will examine the $(1+1)$-dimensional Boussinesq equation [28]:

$$
q_{t t}-q_{x x}+\alpha\left(q^{2}\right)_{x x}-\lambda q_{x x x x}=0, \quad \alpha \neq 0,
$$

which is completely integrable and possesses an infinite number of conservation laws, consequently, permits multiple soliton solutions. It was presented by Boussinesq to pronounce the two-dimensional propagation of shallowwater waves with small amplitude as they propagate at a uniform speed in a canal of constant depth. 
Many variants of the Boussinesq equation were inferred to describe physical phenomena [32-35]. In the present work we will extend our study to the two higher dimensional Boussinesq equations, namely, the $(2+1)$ dimensional Boussinesq equation [29, 30, 36, 37]:

$$
\begin{aligned}
& q_{t t}-a q_{x x}-b q_{y y}+\alpha\left(q^{2}\right)_{x x}-\lambda q_{x x x x}=0, \\
& \alpha \neq 0,
\end{aligned}
$$

and the $(3+1)$-dimensional Boussinesq equation [31]:

$$
\begin{aligned}
& q_{t t}-a q_{x x}-b q_{y y}-c q_{z z}+\alpha\left(q^{2}\right)_{x x}-\lambda q_{x x x x}=0, \\
& \quad \alpha \neq 0 .
\end{aligned}
$$

The equations with $\lambda= \pm 1$ are well-recognized as the good and bad Boussinesq equations in literature for \pm sign, respectively. Equation (2) describes the propagation of gravity waves on the surface of water and in specific the head-on collision of oblique waves [29]. Equations (2) and (3) combine the two-way propagation of the classical Boussinesq equation with the weak dependence on a second variable $y$ and two other new spatial variables $y$ and $z$, respectively.

This paper is organized as follows. In Sects. 2, 3 and 4 we will address the analytical solitary waves and shock wave solutions of $(1+1),(2+1)$ and $(3+1)$-dimensional Boussinesq equations, respectively. In Sect. 5, we will show the effect of the varying parameters on the evolution of solitary waves and shock wave solutions by direct numerical simulation technique. Section 6 will reveal our conclusions.

\section{The $(1+1)$-dimensional Boussinesq equation}

In this section, we will develop the analytical solitary waves and shock wave solutions of $(1+1)$-dimensional Boussinesq equation by method of undetermined coefficients.

\subsection{The solitary wave solution}

We instigate our study by analysing the $(1+1)$ dimensional Boussinesq equation which is given as

$$
q_{t t}-q_{x x}+\alpha\left(q^{2}\right)_{x x}-\lambda q_{x x x x}=0,
$$

where $\alpha$ and $\lambda$ are constants. The solitary wave is regarded as a localized intensity peak above a continuous wave background. In order to find the solitary wave solution for Eq. (4), we practice the following solitary wave form [14-24]

$$
q(x, t)=A \operatorname{sech}^{p}\{\eta(x-v t)\},
$$

where $A, \eta$ and $v$ are respectively, the amplitude, the inverse width and the velocity of the soliton that will be determined as functions of the model coefficients $\alpha$ and $\lambda$. The unknown index $p$, where $p>0$, will be determined during the course of study of the solution of Eq. (4).

Substituting Eq. (5) into Eq. (4), we obtain

$$
\begin{aligned}
& A p^{2} \eta^{2} v^{2} \operatorname{sech}^{p} \theta-p(p+1) A \eta^{2} v^{2} \operatorname{sech}^{p+2} \theta \\
& \quad-A p^{2} \eta^{2} \operatorname{sech}^{p} \theta+p(p+1) A \eta^{2} \operatorname{sech}^{p+2} \theta \\
& \quad+\alpha\left[4 A^{2} \eta^{2} p^{2} \operatorname{sech}^{2 p} \theta-2 p(2 p+1) A^{2} \eta^{2} \operatorname{sech}^{2 p+2} \theta\right] \\
& \quad-\lambda\left[A p^{4} \eta^{4} \operatorname{sech}^{p} \theta\right. \\
& \quad+2 A \eta^{4} p(p+1)\left(p^{2}+2 p+2\right) \operatorname{sech}^{p+2} \theta
\end{aligned}
$$

$$
\left.-A \eta^{4} p(p+1)(p+2)(p+3) \operatorname{sech}^{p+4} \theta\right]=0,
$$

where $\theta=\eta(x-v t)$.

On equating the highest exponents of $\operatorname{sech}^{p+4} \theta$ and $\operatorname{sech}^{2 p+2} \theta$ functions in Eq. (6), one gets

$2 p+2=p+4$,

which employ

$$
p=2 \text {. }
$$

From Eq. (6) setting the coefficients of the same exponent of $\operatorname{sech}^{p+j} \theta$, to zero where $j=0,2,4$, since these are linearly independent functions, gives a set of algebraic equations:

$$
\begin{aligned}
& v^{2}-4 \lambda \eta^{2}-1=0, \\
& 6\left(1-v^{2}\right)+16 \alpha A+120 \lambda A \eta^{2}=0, \\
& -20 \alpha A-120 \lambda A \eta^{2}=0 .
\end{aligned}
$$

From Eq. (9), we obtain

$$
v= \pm \sqrt{4 \lambda \eta^{2}+1}
$$

Solving Eq. (11) using Eq. (10), we obtain

$$
A=\frac{3}{2} \frac{\left(1-v^{2}\right)}{\alpha}=\frac{-6 \lambda \eta^{2}}{\alpha} \text {. }
$$

Now Eq. (13) imposes the constraint conditions on the parameters as $v \neq 1$ and $\alpha \lambda<0$ for the soliton to exist. Equation (12) shows that velocity $v$ is dependent on $\lambda$ and $\eta$ and \pm sign in the soliton velocity shows the chirality of solitary wave solution. The soliton can move in either $+x$ or $-x$ direction, respectively. Finally, we get the solitary wave solution for the $(1+1)$-dimensional Boussinesq equation, when the above expressions of $A$ and $v$ given by Eqs. (12) and (13) are substituted in Eq. (5) as

$$
q(x, t)=\frac{-6 \lambda \eta^{2}}{\alpha} \operatorname{sech}^{2}\{\eta(x-v t)\} .
$$

where the relation between amplitude $A$ and the inverse width $\eta$ of the soliton is depicted by Eq. (13). The soliton velocity $v$ is given by Eq. (12) and the constraint relation assures the existence of the soliton.
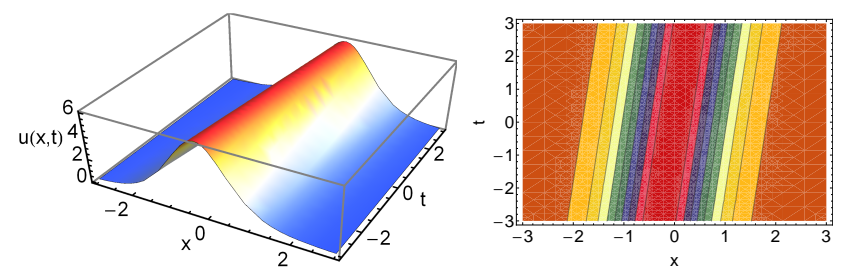

Fig. 1. Profile of the solitary wave solution (14) for parameter values $\alpha=-1, \eta=1, v=2.2360, \lambda=1$.

Figure 1 shows the numerical simulation of the solution with the choice of parameters as $\alpha=-1, \eta=1, v=$ $2.2360, \lambda=1$. This prescribes set of parameter values, which, satisfying the constraint relations, are selected in order to carry out numerical simulation.

\subsection{The shock waves solution}

The shock wave is characterized by a localized drop of intensity related to a more intense continuous wave background. The focus will be on searching the shock wave solution to Eq. (4). In order to seek for shock wave 
solution to Eq. (4), the preliminary assumption is

$$
q(x, t)=A \tanh ^{p}\{\eta(x-v t)\},
$$

where $A, \eta$ are free unknown parameters and $v$ is the velocity of the soliton that will be determined as functions of the model coefficients $\alpha$ and $\lambda$. Additionally, the values of the unknown exponents $p$ will fall out during the course of derivation of the soliton solution.

Substituting Eq. (15) into Eq. (4), we get

$$
\begin{aligned}
& A p v^{2} \eta^{2}\left[(p-1) \tanh ^{p-2} \theta\right. \\
& \left.\quad-2 \tanh ^{p} \theta+(p+1) \tanh ^{p+2} \theta\right] \\
& \quad-A p \eta^{2}\left[(p-1) \tanh ^{p-2} \theta\right. \\
& \left.\quad-2 \tanh ^{p} \theta+(p+1) \tanh ^{p+2} \theta\right] \\
& \quad+2 \alpha A^{2} p \eta^{2}\left[(2 p-1) \tanh ^{2 p-2} \theta\right. \\
& \left.\quad+(2 p+1) \tanh ^{2 p+2} \theta-4 p \tanh ^{2 p} \theta\right] \\
& \quad+2 A^{2} p \eta_{1}^{2}\left[\tanh ^{2 p+2} \theta-\tanh ^{2 p-2} \theta\right] \\
& \quad-\lambda A \eta^{4} p\left[(p+1)(p+2)(p+3) \tanh ^{p+4} \theta\right. \\
& \quad-(p+1)\left(3 p^{2}+3 p+2\right) \tanh ^{p+2} \theta \\
& \quad+(p-1)\left(3 p^{2}-3 p+2\right) \tanh ^{p} \theta \\
& \quad-(p-1)(p-2)(p-3) \tanh ^{p-2} \theta \\
& \quad-(p+1)(p+2)(p+3) \tanh ^{p+2} \theta \\
& \quad+(p+1)\left(3 p^{2}+3 p+2\right) \tanh ^{p} \theta \\
& \quad-(p-1)\left(3 p^{2}-3 p+2\right) \tanh ^{p-2} \theta \\
& \left.\quad+(p-1)(p-2)(p-3) \tanh ^{p-4} \theta\right]=0
\end{aligned}
$$

where $\theta=\eta(x-v t)$.

By equating the highest exponents of $\tanh ^{p+4} \theta$ and $\tanh ^{2 p+2} \theta$ functions in Eq. (16), one obtains

$2 p+2=p+4$,

which leads to

$$
p=2 \text {. }
$$

Collecting the coefficients of the same exponent of $\tanh ^{p} \theta, \tanh ^{p+2} \theta, \tanh ^{2 p-2} \theta, \tanh ^{p+4} \theta$ and $\tanh ^{2 p+2} \theta$, respectively, where each coefficient has to vanish, we obtain the following system of algebraic equations:

$$
\begin{aligned}
& v^{2}-1+8 \lambda \eta^{2}=0, \\
& 1-v^{2}+3 \alpha A+4 \lambda \eta^{2}-30 \lambda \eta^{2}=0, \\
& v^{2}-1-\frac{16}{3} \alpha A+40 \lambda \eta^{2}=0, \\
& \alpha A-6 \lambda \eta^{2}=0 .
\end{aligned}
$$

It is to be noted that the coefficients of the linearly independent functions $\tanh ^{p-4}(\theta)$ in Eq. (16) are spontaneously zero for index $p=2$.

From Eq. (19), the soliton velocity is determined as

$$
v= \pm \sqrt{1-8 \lambda \eta^{2}} \text {. }
$$

Equation (22), leads to

$$
A=\frac{6 \lambda \eta^{2}}{\alpha} \text {. }
$$

Note that by substituting the value of $v$ from Eq. (23) in Eqs. (20) and (21) results in the same expression of free parameter $A$ as given by Eq. (24). Hence these two equations can be understood as integrability conditions for the shock wave solution to exist. Also the free para- meter $A$ of shock wave solution is affected by the presence of other soliton parameters $\alpha$ and $\lambda$. Finally we get the shock wave solution for the $(1+1)$-dimensional Boussinesq equation, when the above expressions of $A$ and $v$ given by Eqs. (23) and (24) are substituted in Eq. (15) as

$$
q(x, t)=\frac{6 \lambda \eta^{2}}{\alpha} \tanh ^{2}\{\eta(x-v t)\} .
$$
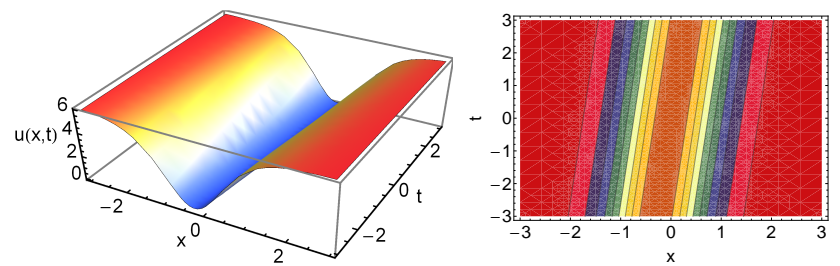

Fig. 2. Profile of the shock wave solution (25) for parameter values $\alpha=1, \eta=0.1, v=0.44, \lambda=1$.

The only other simple condition that needs to hold for the shock wave solution to exist is that $\alpha \lambda>0$ which follows from Eq. (24). From Eqs. (14) and (25), we observed that the existence condition for solitary wave and shock wave solutions are opposite to each other. Figure 2 shows the evolution of the shock wave solution (25) for different model coefficients which satisfy the constraint condition. The choice of parameter for the numerical simulation of the shock wave solution is as $\alpha=1, \eta=0.1, v=0.44, \lambda=1$.

\section{The $(2+1)$-dimensional Boussinesq equation}

In this section, we will reveal the analytical solitary wave and shock wave solutions (domain walls) of $(2+1)$ dimensional Boussinesq equation.

\subsection{The solitary wave solution}

In this subsection, we consider the nonlinear $(2+1)$ dimensional Boussinesq equation

$$
q_{t t}-a q_{x x}-b q_{y y}+\alpha\left(q^{2}\right)_{x x}-\lambda q_{x x x x}=0,
$$

where $a, b, \alpha$ and $\lambda$ are arbitrary constants with $\alpha \neq 0$. This equation would seem to be the prototype for waves that propagate in opposite direction in $(2+1)$ dimensions. Different cases of Eq. (26) are studied in Refs. [29, 30, 36, 37]. When $a=b=\alpha=1$, and $\lambda=-3$, Eq. (26) can be used to define the propagation of gravity waves on the water surface whose periodic solutions, multi-soliton solutions and soliton resonance phenomena have been established in Refs. [15, 16]. To initiate with, the hypothesis is given by

$$
q(x, y, t)=A \operatorname{sech}^{p}\left\{\eta_{1} x+\eta_{2} y-v t\right\} .
$$

Here in Eq. (27) $A, \eta_{1}, \eta_{2}$, and $v$ are, respectively, the amplitude, the inverse widths in $x, y$ directions and the velocity of the wave that will be determined as functions of the model parameters $\alpha$ and $\lambda$. It is to be noted that in the supposition of the solution structure, the inverse widths of the soliton in the $x$ - and $y$-directions are taken to be different, namely $\eta_{1} \neq \eta_{2}$ in common. This makes 
the structure of the soliton solution more generalized. The exponent $p$ will be also determined.

Inserting Eq. (27) into Eq. (26), yields

$$
\begin{aligned}
& A p^{2} v^{2} \operatorname{sech}^{p} \theta-p(p+1) A v^{2} \operatorname{sech}^{p+2} \theta \\
& \quad-A p^{2}\left(a \eta_{1}^{2}+b \eta_{2}^{2}\right) \operatorname{sech}^{p} \theta \\
& \quad+p(p+1) A v^{2}\left(a \eta_{1}^{2}+b \eta_{2}^{2}\right) \operatorname{sech}^{p+2} \theta \\
& \quad+\alpha\left[4 A^{2} \eta_{1}^{2} p^{2} \operatorname{sech}^{2 p} \theta-2 p(2 p+1) A^{2} \eta_{1}^{2} \operatorname{sech}^{2 p+2} \theta\right] \\
& \quad-\lambda\left[A p^{4} \eta_{1}^{4} \operatorname{sech}^{p} \theta\right. \\
& \quad-2 A \eta_{1}^{4} p(p+1)\left(p^{2}+2 p+2\right) \operatorname{sech}^{p+2} \theta \\
& \left.\quad+A \eta_{1}^{4} p(p+1)(p+2)(p+3) \operatorname{sech}^{p+4} \theta\right]=0
\end{aligned}
$$

where $\theta=\eta(x-v t)$.

Equating the highest exponents of $\operatorname{sech}^{p+4} \theta$ and $\operatorname{sech}^{2 p+2} \theta$ functions in Eq. (28), we get

$$
2 p+2=p+4,
$$

which yields

$$
p=2 \text {. }
$$

Now, noting that the functions $\operatorname{sech}^{p} \theta, \operatorname{sech}^{p+2} \theta$, $\operatorname{sech}^{p+4} \theta$, and $\operatorname{sech}^{2 p+2} \theta$ are linearly independent, setting their respective coefficients in Eq. (28) to zero yields the following equations:

$$
\begin{aligned}
& v^{2}-4 \lambda \eta_{1}^{2}-\left(a \eta_{1}^{2}+b \eta_{2}^{2}\right)=0, \\
& 6 v^{2}\left(a \eta_{1}^{2}+b \eta_{2}^{2}-1\right)+16 \alpha A \eta_{1}^{2}+120 \lambda \eta_{1}^{4}=0, \\
& -20 \alpha A \eta_{1}^{2}-120 \lambda \eta_{1}^{4}=0 .
\end{aligned}
$$

From Eq. (31), we get

$$
v= \pm \sqrt{4 \lambda \eta_{1}^{4}+b \eta_{2}^{2}+a \eta_{1}^{2}} .
$$

Using Eq. (33) into Eq. (32), the following value of $A$ is obtained:

$$
A=\frac{3 v^{2}}{2} \frac{\left(a \eta_{1}^{2}+b \eta_{2}^{2}-1\right)}{\alpha \eta_{1}^{2}} .
$$

Thus, the solitary wave solution of the the $(2+1)$ dimensional Boussinesq equation is given by

$$
\begin{aligned}
& q(x, y, t)= \\
& \quad \frac{3 v^{2}}{2} \frac{\left(a \eta_{1}^{2}+b \eta_{2}^{2}-1\right)}{\alpha \eta_{1}^{2}} \operatorname{sech}^{2}\left\{\eta_{1} x+\eta_{2} y-v t\right\} .
\end{aligned}
$$

where the amplitude $A$ is related to the inverse widths $\eta_{1}$ and $\eta_{2}$ as given by (35) and the velocity $v$ is given by (34). In view of Eq. (35), we clearly see that solitary wave solution exist provided that $\alpha \eta_{1}^{2} \neq 0$.
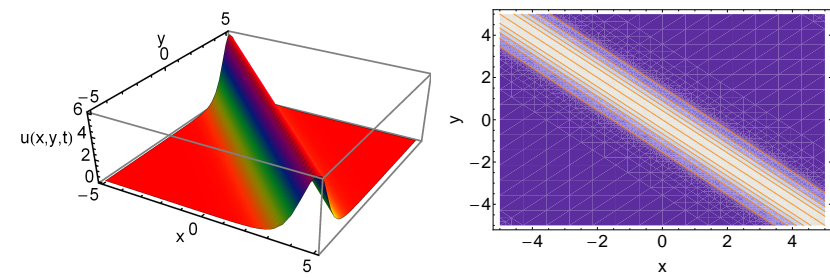

Fig. 3. Profile of the solitary wave solution (35) for parameter values $a=1, b=1, \alpha=1, \eta_{1}=1, \eta_{2}=$ $1, \lambda=1, v=2.4494$, at $t=0$.

Figure 3 shows the profile of a soliton solution of the $(2+1)$-dimensional Boussinesq equation. The parameter values chosen are $a=1, b=1, \alpha=1, \eta_{1}=1, \eta_{2}=1, \lambda=$ $1, v=2.4494$, at $t=0$.

\subsection{The domain wall solution}

In dimensions higher than $(1+1)$ the shock waves or topological solitons are called "domain walls". Now we are interested in finding the domain walls solution for considered $(2+1)$-dimensional Boussinesq equation. To do this, we consider the solution of the form

$$
q(x, y, t)=A \tanh ^{p} \theta,
$$

where $\theta=\left\{\eta_{1} x+\eta_{2} y-v t\right\}$ represents the shape of pulse and $A, \eta_{1}, \eta_{2}$ are unknown free parameters and the $v$ is the velocity of the soliton that will be find out. The exponent $p$ is also unknown.

Substituting Eq. (37) into Eq. (26), we get

$$
\begin{aligned}
& A p^{2} v^{2}\left[\tanh ^{p-2} \theta-2 \tanh ^{p} \theta+\tanh ^{p+2} \theta\right] \\
& \quad+A p v^{2}\left[\tanh ^{p+2} \theta-\tanh ^{p-2} \theta\right] \\
& \quad-p A\left(a \eta_{1}^{2}+b \eta_{2}^{2}\right)\left\{(p-1) \tanh ^{p-2} \theta\right. \\
& \left.\quad-2 p \tanh ^{p} \theta+(p+1) \tanh ^{p+2} \theta\right\} \\
& \quad+\alpha\left[4 A^{2} p^{2} \eta_{1}^{2}\left\{\tanh ^{2 p-2} \theta+\tanh ^{2 p+2} \theta-2 \tanh ^{2 p} \theta\right\}\right. \\
& \left.\quad+2 A^{2} p \eta_{1}^{2}\left\{\tanh ^{2 p+2} \theta-\tanh ^{2 p-2} \theta\right\}\right] \\
& \quad-\lambda\left[p A \eta _ { 1 } ^ { 4 } \left\{(p-1)(p-2)(p-3) \tanh ^{p-4} \theta\right.\right. \\
& \quad-4(p-1)\left(p^{2}-2 p+2\right) \tanh ^{p-2} \theta \\
& \quad+2 p\left(3 p^{2}+5\right) \tanh ^{p} \theta \\
& \quad-4(p+1)\left(p^{2}+2 p+2\right) \tanh ^{p+2} \theta \\
& \left.\left.\quad+(p+1)(p+2)(p+3) \tanh ^{p+4} \theta\right\}\right]=0 .
\end{aligned}
$$

From Eq. (38), equating the highest exponents of $\tanh ^{p+4} \theta$ and $\tanh ^{2 p+2} \theta$ functions, we have

$2 p+2=p+4$,

which provides

$$
p=2 \text {. }
$$

Collecting the coefficients of the same exponent of $\tanh ^{p} \theta, \tanh ^{p+2} \theta, \tanh ^{2 p-2} \theta, \tanh ^{p+4} \theta$, and $\tanh ^{2 p+2} \theta$, respectively, where each coefficient has to vanish, we obtain the following system of algebraic equations:

$$
\begin{aligned}
& v^{2}-\left(a \eta_{1}^{2}+b \eta_{2}^{2}\right)+8 \lambda \eta_{1}^{4}=0, \\
& -v^{2}+\left(a \eta_{1}^{2}+b \eta_{2}^{2}\right)-\frac{3}{2} \alpha A \eta_{1}^{2}-17 \lambda \eta_{1}^{4}=0, \\
& v^{2}-\left(a \eta_{1}^{2}+b \eta_{2}^{2}\right)-\frac{16}{3} \alpha A \eta_{1}^{2}+40 \lambda \eta_{1}^{4}=0, \\
& \alpha A+6 \lambda \eta_{1}^{2}=0 .
\end{aligned}
$$

From Eq. (41), one gets

$$
v= \pm \sqrt{b \eta_{2}^{2}+a \eta_{1}^{2}-8 \lambda \eta_{1}^{4}} .
$$

Now solving Eq. (44), we get

$$
A=\frac{-6 \lambda \eta_{1}^{2}}{\alpha} \text {. }
$$

From Eq. (46), we clearly see that this solution exists provided $\alpha \lambda<0$ and the free parameter $A$ only depends on $\eta_{1}$, independent of $\eta_{2}$ and $\eta_{3}$. Note that by substituting the value of $v$ from Eq. (45) in Eqs. (42) and (43) results in the same expression of $A$ as given by (46). Hence these equations can be understood as integrability condition for the domain walls. We would like to note that 
for the \pm sign in the expression (45), the domain walls either move in left (for - sign) or right (for + sign) hand directions, respectively. Thus, the domain walls solution of the $(2+1)$-dimensional Boussinesq equation is given by

$$
\left.q(x, y, t)=\frac{-6 \lambda \eta_{1}^{2}}{\alpha} \tanh ^{2}\left\{\eta_{1} x+\eta_{2} y-v t\right)\right\},
$$
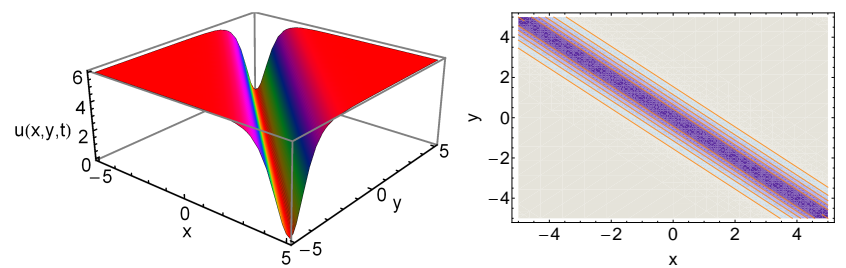

Fig. 4. Profile of the domain wall solution (47) for parameter values $\alpha=-1, \eta_{1}=1, \eta_{2}=1, t=0, \lambda=1$.
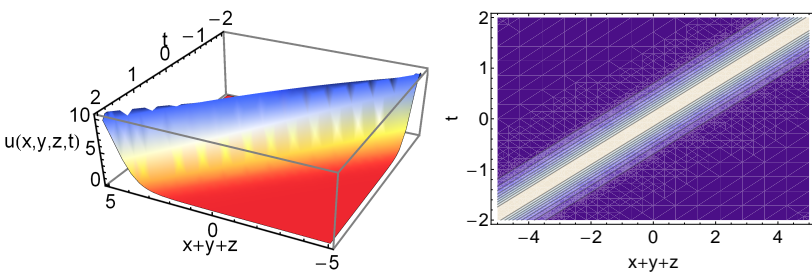

Fig. 5. Profile of the soliton solution for parameter values $a=b=c=1, \alpha=1, \eta_{1}=1, \eta_{2}=1, \eta_{3}=1, v=$ $2.6457, \lambda=1$.
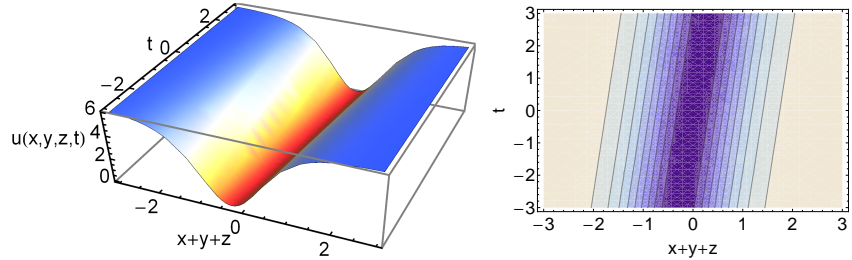

Fig. 6. Profile of the domain wall solution for parameter values $\alpha=-1, \eta_{1}=1, \eta_{2}=1, \eta_{3}=1, a=b=4, c=$ $1, v=1, \lambda=1$.

where the velocity of the domain walls is given by (45) and the free parameters relation of the domain walls is specified by (46). Figure 4 shows the profile of the domain wall solution of the $(2+1)$-dimensional Boussinesq equation. The parameter values chosen are $\alpha=-1, \eta_{1}=$ $1, \eta_{2}=1, \lambda=1$ at $t=0$.

\section{The $(3+1)$-dimensional Boussinesq equation}

In this section, we will utilize the method of undetermined coefficients to solve the $(3+1)$-dimensional Boussinesq equation.

\subsection{The solitary wave solution}

In this subsection, the search is going for finding the solitary wave solution, for the $(3+1)$-dimensional Boussinesq equation which is considered as

$q_{t t}-a q_{x x}-b q_{y y}-c q_{z z}+\alpha\left(q^{2}\right)_{x x}-\lambda q_{x x x x}=0$, (48) which can be generalized from Eq. (26), with $c$ as the coefficient of dissipation term in the $z$ direction. We know that the $(3+1)$-dimensional Boussinesq equation does not have the three-soliton solution and this implies that none of the $(3+1)$-dimensional Boussinesq equations should be integrable, and partially clarify why it does not pass the Painlevé integrability test.

In order to look for solitary wave solution to Eq. (48), we exercise the following wave form

$$
q(x, y, z, t)=A \operatorname{sech}^{p} \theta,
$$

where $\theta=\left\{\eta_{1} x+\eta_{2} y+\eta_{3} z-v t\right\}$, where in Eq. (49), the parameters $A, \eta_{1}, \eta_{2}, \eta_{3}$ are known as the amplitude and soliton inverse widths in $x, y$ and $z$ directions respectively for soliton and $v$ is the velocity of the soliton. Note that in the sense of extracting the solitary wave solution for Eq. (48), one needs to have $p>0$ for soliton to exist.

Substituting Eq. (49) into Eq. (48), leads to

$$
\begin{aligned}
& A p^{2} v^{2} \operatorname{sech}^{p} \theta-p(p+1) A v^{2} \operatorname{sech}^{p+2} \theta \\
& \quad-A p^{2}\left(a \eta_{1}^{2}+b \eta_{2}^{2}+c \eta_{3}^{2}\right) \operatorname{sech}^{p} \theta \\
& \quad+p(p+1) A v^{2}\left(a \eta_{1}^{2}+b \eta_{2}^{2}\right) \operatorname{sech}^{p+2} \theta \\
& \quad+\alpha\left[4 A^{2} \eta_{1}^{2} p^{2} \operatorname{sech}^{2 p} \theta\right. \\
& \left.\quad-2 p(2 p+1) A^{2} \eta_{1}^{2} \operatorname{sech}^{2 p+2} \theta\right] \\
& \quad-\lambda\left[A p^{4} \eta_{1}^{4} \operatorname{sech}^{p} \theta\right. \\
& \quad-2 A \eta_{1}^{4} p(p+1)\left(p^{2}+2 p+2\right) \operatorname{sech}^{p+2} \theta \\
& \left.\quad+A \eta_{1}^{4} p(p+1)(p+2)(p+3) \operatorname{sech}^{p+4} \theta\right]=0 .
\end{aligned}
$$

From Eq. (50) equating the exponents of $\operatorname{sech}^{p+4} \theta$ and $\operatorname{sech}^{2 p+2} \theta$ functions, we set

$$
2 p+2=p+4,
$$

so that

$$
p=2 \text {. }
$$

By setting the corresponding coefficients of $\operatorname{sech}^{p} \theta$, $\operatorname{sech}^{p+2} \theta, \operatorname{sech}^{p+4} \theta$ and $\operatorname{sech}^{2 p+2} \theta$ to zero, we obtain the following system of algebraic equations:

$$
\begin{aligned}
& v^{2}-4 \lambda \eta_{1}^{2}-\left(a \eta_{1}^{2}+b \eta_{2}^{2}\right)=0, \\
& 6 v^{2}\left(a \eta_{1}^{2}+b \eta_{2}^{2}+c \eta_{3}^{2}-1\right)+16 \alpha A \eta_{1}^{2} \\
& \quad+120 \lambda \eta_{1}^{4}=0, \\
& \quad-20 \alpha A \eta_{1}^{2}-120 \lambda \eta_{1}^{4}=0 .
\end{aligned}
$$

From Eq. (53), we have

$$
v= \pm \sqrt{4 \lambda \eta_{1}^{4}+a \eta_{1}^{2}+b \eta_{2}^{2}+c \eta_{3}^{2}} .
$$

Using Eq. (55) into Eq. (54), we obtain

$$
A=\frac{3 v^{2}}{2} \frac{\left(a \eta_{1}^{2}+b \eta_{2}^{2}+c \eta_{3}^{2}-1\right)}{\alpha \eta_{1}^{2}} .
$$

The latter shows that solitons exist for $\alpha \eta_{1}^{2} \neq 0$. Thus, finally, soliton solution to $(3+1)$-dimensional Boussinesq equation given by Eq. (48) with constant coefficients is

$$
\begin{aligned}
& q(x, y, z, t)=\frac{3 v^{2}}{2} \frac{\left(a \eta_{1}^{2}+b \eta_{2}^{2}+c \eta_{3}^{2}-1\right)}{\alpha \eta_{1}^{2}} \\
& \quad \times \operatorname{sech}^{2}\left\{\eta_{1} x+\eta_{2} y+\eta_{3} z-v t\right\},
\end{aligned}
$$

where velocity of soliton is given by Eq. (56) and amplitude $A$ of the soliton is seen in Eq. (57). It is necessary to observe that the amplitude $A$ is dependent on the soliton inverse widths $\eta_{1}, \eta_{2}, \eta_{3}$ and velocity 
of the soliton $v$. Therefore, the choice of model parameters $a, b, c, \alpha$ and $\lambda$ directly affects the amplitude of shock wave solution. Figure 5 represents the 3-dimensional surface plots of the solitary wave solution (58) for different parameter values given as $a=b=c=1$, $\alpha=1, \eta_{1}=1, \eta_{2}=1, \eta_{3}=1, v=2.6457, \lambda=1$.

\subsection{The domain wall solution}

In order to construct domain wall solutions for Eq. (48), we assume, the solution of the form

$$
q(x, y, z, t)=A \tanh ^{p} \theta
$$

and choose now a suitable wave form with $(3+1)$ dependent variables of the form $\theta=\left\{\eta_{1} x+\eta_{2} y+\eta_{3} z-v t\right\}$. For domain walls, the parameters $A, \eta_{1}, \eta_{2}, \eta_{3}$ are indeed free domain wall parameters while $v$ still represents the velocity of the domain walls, that will be determined. The exponent $p$ is also unknown and determined during the fall out of domain wall derivation. Substituting Eq. (59) into Eq. (48), we get

$$
\begin{aligned}
& A p^{2} v^{2}\left[\tanh ^{p-2} \theta-2 \tanh ^{p} \theta+\tanh ^{p+2} \theta\right] \\
& \quad+A p v^{2}\left[\tanh ^{p+2} \theta-\tanh ^{p-2} \theta\right] \\
& \quad-p A\left(a \eta_{1}^{2}+b \eta_{2}^{2}+c \eta_{3}^{2}\right)\left\{(p-1) \tanh ^{p-2} \theta\right. \\
& \left.\quad-2 p \tanh ^{p} \theta+(p+1) \tanh ^{p+2} \theta\right\} \\
& \quad+\alpha\left[4 A^{2} p^{2} \eta_{1}^{2}\left\{\tanh ^{2 p-2} \theta+\tanh ^{2 p+2} \theta-2 \tanh ^{2 p} \theta\right\}\right. \\
& \left.\quad+2 A^{2} p \eta_{1}^{2}\left\{\tanh ^{2 p+2} \theta-\tanh ^{2 p-2} \theta\right\}\right] \\
& \quad-\lambda\left[p A \eta _ { 1 } ^ { 4 } \left\{(p-1)(p-2)(p-3) \tanh ^{p-4} \theta\right.\right. \\
& \quad-4(p-1)\left(p^{2}-2 p+2\right) \tanh ^{p-2} \theta \\
& \quad+2 p\left(3 p^{2}+5\right) \tanh ^{p} \theta \\
& \quad-4(p+1)\left(p^{2}+2 p+2\right) \tanh ^{p+2} \theta \\
& \left.\left.\quad+(p+1)(p+2)(p+3) \tanh ^{p+4} \theta\right\}\right]=0,
\end{aligned}
$$

where $\theta=\eta_{1} x+\eta_{2} y+\eta_{3} z-v t$.

Thus, for matching the highest exponents of $\tanh ^{p+4} \theta$ and $\tanh ^{2 p+2} \theta$ functions in Eq. (60), one gets

$2 p+2=p+4$,

which yields

$$
p=2 \text {. }
$$

Collecting the coefficients of the same exponent of $\tanh ^{p} \theta, \tanh ^{p+2} \theta, \tanh ^{2 p-2} \theta, \tanh ^{p+4} \theta$ and $\tanh ^{2 p+2} \theta$, respectively, where each coefficient has to vanish, we obtain the following system of algebraic equations:

$$
\begin{aligned}
& v^{2}-\left(a \eta_{1}^{2}+b \eta_{2}^{2}+c \eta_{3}^{2}\right)+8 \lambda \eta_{1}^{4}=0, \\
& -v^{2}+\left(a \eta_{1}^{2}+b \eta_{2}^{2}+c \eta_{3}^{2}\right)-\frac{3}{2} \alpha A \eta_{1}^{2}-17 \lambda \eta_{1}^{4}=0, \\
& v^{2}-\left(a \eta_{1}^{2}+b \eta_{2}^{2}+c \eta_{3}^{2}\right)-\frac{16}{3} \alpha A \eta_{1}^{2}+40 \lambda \eta_{1}^{4}=0, \\
& \alpha A+6 \lambda \eta_{1}^{2}=0 .
\end{aligned}
$$

From Eq. (63), one gets

$$
v= \pm \sqrt{a \eta_{3}^{2}+b \eta_{2}^{2}+c \eta_{1}^{2}-8 \lambda \eta_{1}^{4}} .
$$

Equation (67) leads to

$$
A=\frac{-6 \lambda \eta_{1}^{2}}{\alpha}
$$

Also, substituting the value of $v$ from Eq. (67) in Eqs. (64) and (65), one gets the same expression of $A$ as given in (68). From Eqs. (67) and (68), it has been seen that the velocity of domain wall depends on the free parameters $\eta_{1}, \eta_{2}$ and $\eta_{3}$ of soliton in $x, y$ and $z$ directions while the free parameter $A$ of domain walls depends on $\alpha, \lambda$ and $\eta_{1}$. The constraint relation between the coefficients is given by $\alpha \lambda<0$, which must hold for the domain walls to exist.

Lastly, we can determine the domain wall solution for for the $(3+1)$-dimensional Boussinesq equation as

$$
\begin{aligned}
& q(x, y, z, t)= \\
& \quad \frac{-6 \lambda \eta_{1}^{2}}{\alpha} \tanh ^{2}\left\{\eta_{1} x+\eta_{2} y+\eta_{3} z-v t\right\},
\end{aligned}
$$

where the velocity of shock wave is given by Eq. (67) and free parameter $A$ is given by (68). Note that the formation condition of solitary wave and domain wall pulses are opposite to each other. Figure 6 represents the 3 -dimensional surface plots of the domain wall solution (69) for different parameter values given as $\alpha=-1, \eta_{1}=$ $1, \eta_{2}=1, \eta_{3}=1, a=b=4, c=1, v=1, \lambda=1$.

\section{Numerical analysis}

The numerical analysis is necessary for seeking the evolution of solitary wave and shock wave solutions. Therefore we select the direct numerical simulation method for solving the Boussinesq equation. To study the effect of parameters on travelling waves, we consider the following two cases for different values of parameters as:

Case(1): We study the effect of changing the parameter $\lambda, \alpha$ and $\eta$, on evolution of solitary wave (14), namely, three cases arise:
(i) $\alpha=-1, \eta=1, \lambda=1,2,3$.
(ii) $\alpha=-1,-2,-3, \eta=1, \lambda=1$.
(iii) $\alpha=-1, \eta=1,2,3, \lambda=1$.
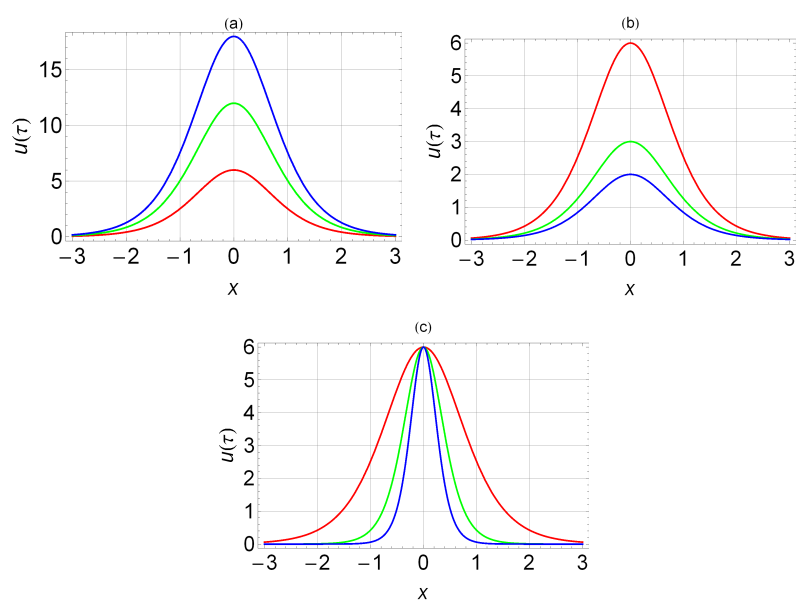

Fig. 7. Profile of the solitary wave solution (14) for parameter values: (a) $\alpha=-1, \eta=1, \lambda=1,2,3$ (red, green, blue), (b) $\alpha=-1,-2,-3$ (red, green, blue), $\eta=$ $1, \lambda=1,(\mathrm{c}) \alpha=-1, \eta=1,2,3$ (red, green, blue), $\lambda=1$.

Figure 7 shows the effect of parameter change on the solitary waves (14). From Fig. 7a, we note that with increase in value of $\lambda$, there is a corresponding increase in 

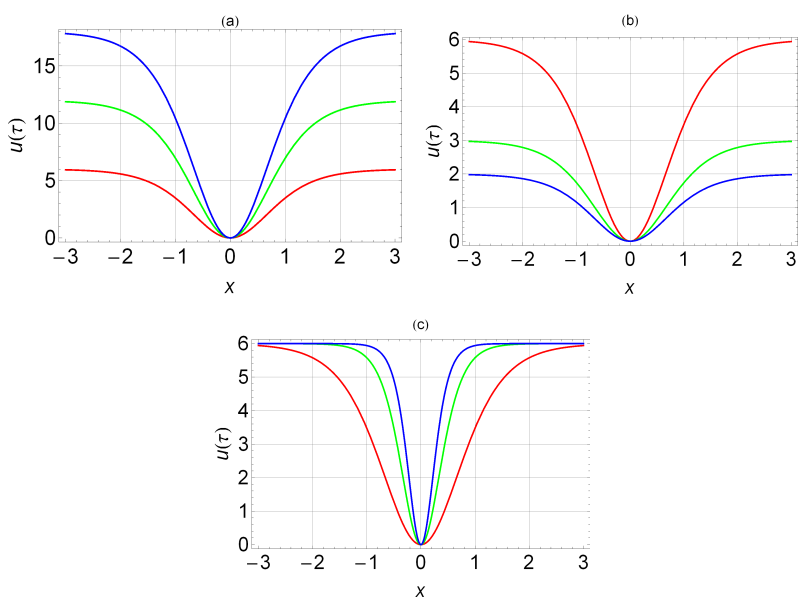

Fig. 8. Profile of the shock wave solution (25) for parameter values: (a) $\alpha=1, \eta=1, \lambda=1,2,3$ (red, green, blue), (b) $\alpha=1,2,3$ (red, green, blue), $\eta=1, \lambda=1$, (c) $\alpha=1, \eta=1,2,3$ (red, green, blue), $\lambda=1$.

amplitude of the solitary wave while Fig. 7b shows that with increase in negative value of $\alpha$, there is a corresponding decrease in amplitude of the solitary wave solution. Hence the presence of parameters $\alpha$ and $\lambda$ directly affects the amplitude of solitons. For constant $\alpha$ and $\lambda$, the increase in value of inverse width parameter $\eta$ will result in the narrower width of soliton as shown in Fig. 7c.

Case(2): We consider the effect of changing the parameter $\lambda, \alpha$ and $\eta$, on evolution of shock wave solution (25).Generally, three cases arise:

(i) $\alpha=1, \eta=1, \lambda=1,2,3$.

(ii) $\alpha=1,2,3, \eta=1, \lambda=1$.

(iii) $\alpha=1, \eta=1,2,3, \lambda=1$.

In Fig. 8 we show the effect of the parameters change on waveform of shock wave solution. From Fig. 8a we conclude that with increase in value of $\lambda$, the amplitude of shock wave increases. From Fig. 8b we note that with increase in value of $\alpha$, the amplitude of shock waves decreases. Therefore, the variation of parameters $\alpha$ and $\lambda$ directly affects the amplitude of shock waves. For constant $\alpha$ and $\lambda$, the increase in value of free parameter $\eta$ will decrease in the pulse width of shock waves as shown in Fig. 8c.

\section{Conclusions}

In this work, one such contemporary method of integrability will be applied to carry out the integration of various Boussinesq equations. The technique of undetermined coefficients will be taken on to integrate such equations. The formation conditions for the envelope solitons and domain walls have also been reported. We also shown that the method of undetermined coefficients is an effective and efficient tool for constructing exact solutions for such type of nonlinear evolution equations. To our knowledge, these new solutions have not been reported earlier and they may be of substantial importance for explaining some special physical phenomena. We trust that the present solutions may be useful in further numerical investigation and these results are going to be very significant in future research.

\section{Acknowledgments}

The authors wish to thank the referees for their valuable suggestions and comments on this paper.

\section{References}

[1] L. Debnath, Nonlinear Partial Differential Equations for Scientists and Engineers, Birkhauser, Boston 1997.

[2] M.J. Ablowitz, P.A. Clarkson, Solitons, Nonlinear Evolution Equations and Inverse Scattering Transform, Cambridge Univ. Press, Cambridge 1990.

[3] E.G. Fan, Phys. Lett. A 277, 212 (2000).

[4] H. Kumar, F. Chand, AIP Adv. 3, 032128 (2013).

[5] A.J. Mohamad Jawad, M.D. Petkovic, P. Laketa, A. Biswas, Sci. Iran. Trans. B Mech. Eng. 20, 179 (2013).

[6] H. Kumar, A. Malik, F. Chand, S.C. Mishra, Indian J. Phys. 86, 819 (2012).

[7] H. Kumar, F. Chand, J. Theor. Appl. Phys. 8, 114 (2014).

[8] E.M. Zayed, J. Phys. A Math. Theor. 42, 195202 (2009).

[9] A. Malik, F. Chand, H. Kumar, S.C. Mishra, Pramana J. Phys. 78, 513 (2012).

[10] H. Kumar, A. Malik, F. Chand, J. Math. Phys. 53, 103704 (2012).

[11] A.J.M. Jawad, M.D. Petkovic, A. Biswas, Iran. J. Sci. Tech. Trans. A 37, 109 (2013).

[12] A. Biswas, M.S. Ismail, Appl. Math. Comput. 216, 3662 (2010).

[13] A.J.M. Jawad, M. Mirzazadeh, A. Biswas, Discrete Cont. Dyn. Syst. Ser. S 8, 1155 (2015).

[14] S. El-Ganaini, M. Mirzazadeh, A. Biswas, Appl. Comput. Math. 14, 248 (2015).

[15] G. Ebadi, N. Yousefzadeh, H. Triki, A. Yildirim, A. Biswas, Romanian Rep. Phys. 64, 915 (2012).

[16] H. Kumar, F. Chand, Optik 125, 2938 (2014).

[17] H. Kumar, F. Chand, Opt. Laser Tech. 54, 265 (2013).

[18] E.V. Krishnan, S. Kumar, A. Biswas, Nonlin. Dyn. 70, 1213 (2012).

[19] A.M. Wazwaz, Appl. Math. Comput. 217, 2277 (2010).

[20] H. Kumar, F. Chand, Indian J. Phys. 87, 909 (2013).

[21] H. Triki, A.H. Kara, A. Biswas, Indian J. Phys. 88, 751 (2014).

[22] H. Triki, A.H. Kara, A. Bhrawy, A. Biswas, Acta Phys. Pol. A 125, 1099 (2014).

[23] H. Kumar, A. Malik, F. Chand, Pramana J. Phys. 80, 361 (2013).

[24] H. Kumar, F. Chand, J. Nonlin. Opt. Phys. Mater. 22, 1350001 (2013). 
[25] A. Biswas, Appl. Math. Lett. 22, 1775 (2009).

[26] A.M. Wazwaz, H. Triki, Commun. Nonlin. Sci. Num. Simul. 16, 1122 (2011).

[27] A. Biswas, Phys. Lett. A 372, 4601 (2008).

[28] M. Chen, Appl. Math. Lett. 11, 45 (1998).

[29] R.S. Johnson, J. Fluid Mech. 323, 65 (1996).

[30] J. Zhang, X. Lai, J. Phys. Soc. Jpn. 75, 2402 (2004).

[31] W. Yong, Chin. Phys. Lett. 25, 2739 (2008).

[32] G. Ebadi, S. Johnson, E. Zerrad, A. Biswas, J. King Saud Univ. Sci. 24, 237 (2012).
[33] H. Triki, A. Chowdhury, A. Biswas, Univ. Pol. Bucharest Sci. Bull. Ser. A 75, 39 (2013).

[34] R. Abazari, S. Jamshidzadeh, A. Biswas, Complexity 21, 151 (2016).

[35] S.O. Adesanya, M. Mirzazadeh, M. Eslami, A. Biswas, J. Comput. Theor. Nanosci. 13, 4739 (2016).

[36] Y. Chen, Z.Y. Yan, H.Q. Zhang, Phys. Lett. A 307, 107 (2003).

[37] F. Tascan, A. Bekir, Appl. Math. Comput. 215, 3134 (2009). 